\title{
Rectangle-Visibility Representations of Bipartite Graphs
}

\author{
Extended Abstract
}

\author{
Alice M. Dean \\ Department of Mathematics \\ AND CoMputer ScIEnce \\ SKIDMORE College \\ Saratoga SPRings, NY 12866 \\ E-MAIL: adean@skidmore.edu \\ Joan P. Hutchinson \\ DEPARTMENT OF MATHEMATICS \\ AND Computer Science \\ Macalester College \\ ST. PAUL, MN 55105 \\ E-MAIL: hutchinson@macalstr.edu
}

\begin{abstract}
The paper considers representations of bipartite graphs as rectanglevisibility graphs, i.e., graphs whose vertices are rectangles in the plane, with adjacency determined by horizontal and vertical visibility. It is shown that, for $p \leq q, K_{p, q}$ has a representation with no rectangles having collinear sides if and only if $p<3$ or $p=3$ and $q \leq 4$. More generally, it is shown that $K_{p, q}$ is a rectangle-visibility graph if and only if $p \leq 4$. Finally, it is shown that every bipartite rectangle-visibility graph on $n \geq 4$ vertices has at most $4 n-12$ edges.
\end{abstract}

1. Introduction and Background. A natural representation of graphs is via geometric shapes placed in the plane with adjacencies determined by certain geometric properties such as visibility or distance. For example, bar-visibility graphs (also known as bar-representable or $\epsilon$-visible graphs) are those planar graphs whose vertices can be represented by horizontal line segments with adjacency determined by vertical visibility; examples can be found in [11]. Here we consider rectangle-visibility graphs, where adjacencies among rectangles in the plane are determined by horizontal and vertical visibility. These graphs have obvious application to VLSI design and have been considered in connection with circuit board design in [4,6], for their own sake in [7], and were the subject under consideration at the Workshop on Visibility Representations, McGill University Bellairs Research Institute, February, 1993. In this paper we characterize 
those complete bipartite graphs that can be so represented in general and when rectangles are not allowed to have collinear sides.

We begin by giving more precise definitions. $G$ is a bar-visibility graph if its vertices can be represented by closed horizontal line segments in the plane, pairwise disjoint except possibly for overlapping endpoints, in such a way that two vertices $u$ and $v$ are adjacent if and only if each of the corresponding segments is vertically visible from the other. In other words, there is a non-degenerate rectangle $R_{u, v}$ with two opposite sides that are subsets of each of these segments, and $R_{u, v}$ intersects no other segment. (See $[8,12,13]$ for related definitions and results.) All such graphs are planar, and in [12] and [13] it is shown that a planar graph is a bar visibility graph if and only if it can be drawn in the plane with all cut-vertices on one face.

$G$ is a rectangle-visibility graph if its vertices can be represented by closed rectangles in the plane with sides parallel to the axes, pairwise disjoint except possibly for overlapping boundaries, in such a way that two vertices $u$ and $v$ are adjacent if and only if each of the corresponding rectangles is vertically or horizontally visible from the other. In other words, there is a non-degenerate rectangle $R_{u, v}$ with two opposite sides that are subsets of each of these rectangles, and $R_{u, v}$ intersects no other rectangle. Note that the zones of visibility may intersect and cross one another.

By the thickness of a graph we mean the minimum number of subsets into which its edge set can be partitioned so that the induced graph on each subset is planar. Hence a graph is planar if and only if it has thickness one. As bar-visibility graphs are naturally planar, so rectangle-visibility graphs naturally have thickness at most two. Given a rectangle-visibility layout of a graph, we can partition its edges into two sets corresponding to vertical and horizontal visibilities, each of which induces a planar subgraph (indeed a bar-visible subgraph) of $G$; hence every rectangle-visibility graph has thickness at most two. Kirkpatrick and Wismath [8] have shown that every planar graph is a rectangle-visibility graph. A rectangle-visibility layout for $K_{4, q}$ and the corresponding partition into two planar subgraphs is given in Figure 1.
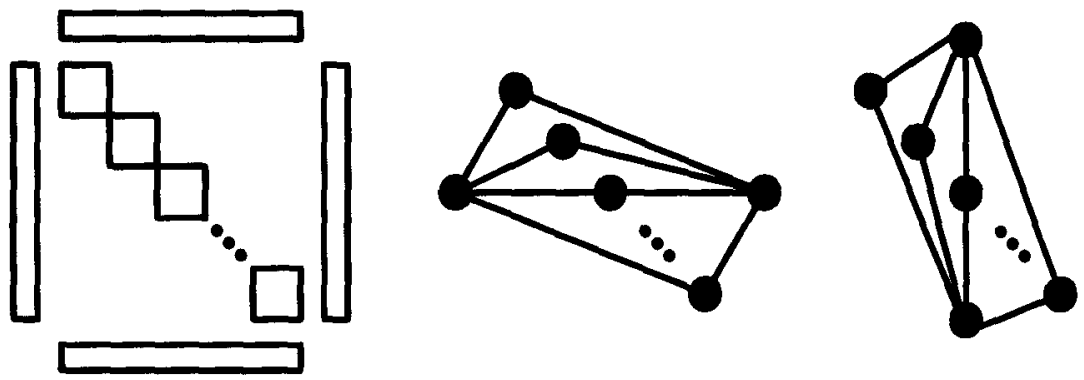

FIgURE 1. A rectangle-visibility layout of $K_{4, q}$ and the corresponding partition of $K_{4, q}$ into two planar subgraphs

By removing external rectangles from the layout of $K_{4, q}$ given in Figure 1, it 
is easy to see that $K_{p, q}$ is a rectangle-visibility graph for $p \leq 4$ and all $q$.

At the Workshop on Visibility Representations, it was conjectured that every rectangle-visibility graph has a layout in which no two rectangles have a pair of collinear sides; call such a representation noncollinear. The layout of $K_{3,4}$ given in Figure 2 has this property. We show that a noncollinear representation is impossible for $K_{4,4}$ and for $K_{p, q}$ with $p \geq 3$ and $q \geq 5$.

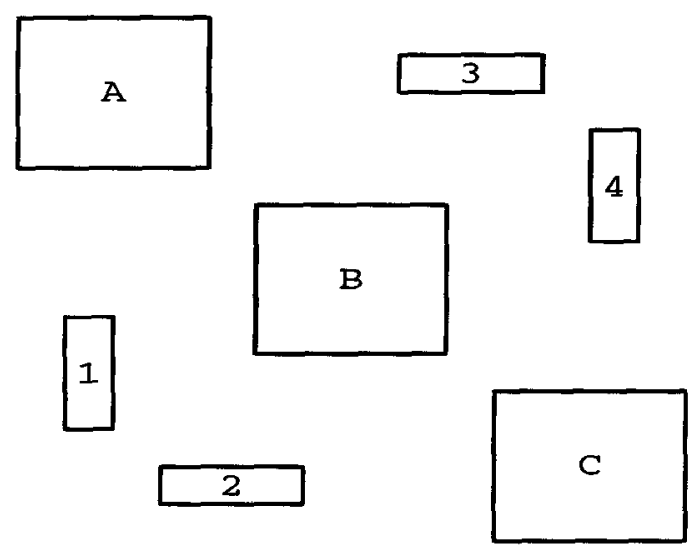

FIGURE 2. A noncollinear rectangle-visibility layout of $K_{3,4}$

It is clear from Figure 1 that $K_{p, q}, p \leq 4$, has thickness at most two. From [2] it is known that the only other thickness-two complete bipartite graphs are $K_{5, q}, q \leq 12$, and $K_{6, q}, q \leq 8$. We prove that $K_{p, q}$ is a rectangle-visibility graph (without concern for noncollinearity) if and only if $p \leq 4$. Table 1 indicates thickness-two complete bipartite graphs, those that are rectangle-visibility graphs, and those that are noncollinear rectangle-visibility graphs.

\begin{tabular}{|c|c|c|c|c|c|c|c|c|c|c|c|c|c|}
\hline $\mathbf{p}$ & 1 & 2 & 3 & 4 & 5 & 6 & 7 & 8 & 9 & 10 & 11 & 12 & $\ldots$ \\
\hline 0 & NR & NR & NR & NR & NR & NR & NR & NR & NR & NR & NR & NR & NR \\
\hline 1 & NR & NR & NR & NR & NR & NR & NR & NR & NR & NR & NR & NR & NR \\
\hline 2 & NR & NR & NR & NR & NR & NR & NR & NR & NR & NR & NR & NR \\
\hline 3 & NR & NR & R & R & R & R & R & R & R & R & R \\
\hline 4 & R & R & R & R & R & R & R & R & R & R \\
\hline 5
\end{tabular}

TABLE 1. Thickness $\leq 2$ (unshaded), rectangle-visibility $(R)$, and noncollinear rectangle-visibility $(N R)$ complete bipartite graphs $K_{p, q}, p \leq q$

The complete and nearly complete bipartite graphs provide a source of examples for distinguishing among various subclasses of the class of thickness-two 
graphs. In [7] $K_{5,5}$ is used to distinguish between the classes of rectanglevisibility graphs and doubly linear graphs, which are those graphs that can be drawn in the plane, using straight lines for edges, in such a way that the edges can be partitioned into two sets inducing plane graphs. $K_{5,5}$ is doubly linear, but it is not a rectangle-visibility graph. Figure 3 shows a rectangle-visibility layout of $K_{5,5}$ minus an edge and Figure 4 a layout of $K_{5,5}$ plus an edge, demonstrating how a small change in a graph can affect its membership in the class of rectangle-visibility graphs. The latter layout also demonstrates that the class of rectangle-visibility graphs is not closed under the operation of taking subgraphs.

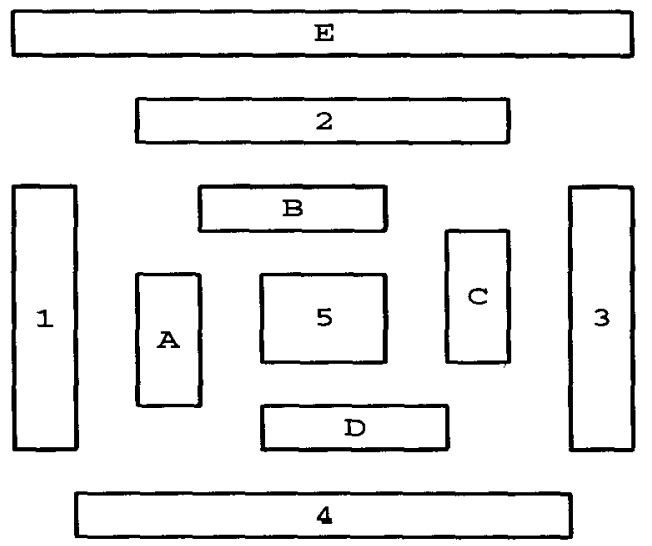

Figure 3. A rectangle-visibility layout of $K_{5,5}$ minus the edge $(E, 5)$

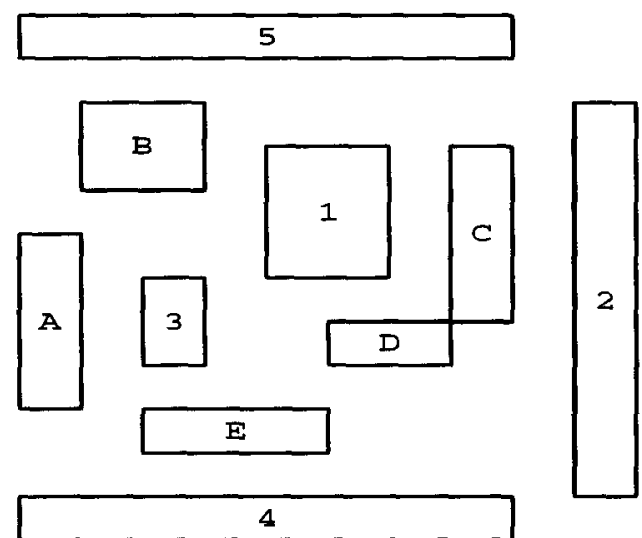

Figure 4. A rectangle-visibility layout of $K_{5,5}$ plus the edge $(1,5)$

Another subclass of thickness-two graphs is the class of strong rectanglevisibility graphs, which permits visibility along degenerate rectangles, i.e., visibility along a line (rectangles now are not permitted to have boundary overlaps). 
The analogous class of strong bar-visibility graphs has been studied in $[1,12]$ and elsewhere. In [12] it is shown that the noncollinear bar-visibility graphs form a strict subclass of the strong bar-visibility graphs, which are in turn a strict subclass of the bar-visibility graphs. The same subclass ordering is easily shown to hold for rectangle-visibility, but it has not been shown to be strict. Figure 5 gives a strong rectangle-visibility layout of $K_{4,4}$ minus an edge that is not a noncollinear layout, and Figures 3 and 4 give rectangle-visibility layouts, respectively, of $K_{5,5}$ plus or minus an edge that are not strong layouts. We conjecture that $K_{4,4}$ minus an edge has no noncollinear layout and that the graphs $K_{5,5}$ plus or minus an edge do not have strong layouts; if true, this would further underscore the usefulness of complete and nearly complete bipartite graphs for distinguishing among several subclasses of thickness-two graphs.

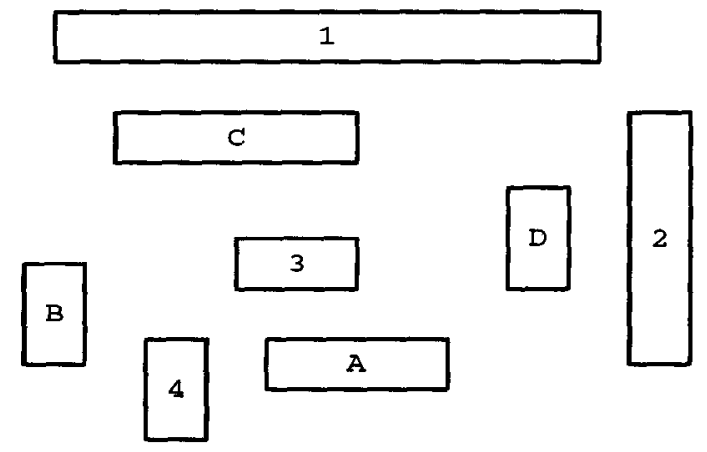

FIGURE 5. A strong rectangle-visibility layout of $K_{4,4}$ minus the edge $(D, 4)$

The fact that not all thickness-two graphs are rectangle-visibility graphs is also shown in [7], where it is proved that a rectangle-visibility graph with $n$ vertices has at most $6 n-20$ edges for $n \geq 5$, and that this bound is best possible for $n \geq 8$. By Euler's Formula, a thickness-two graph can have (and many do have) as many as $6 n-12$ edges. Both the complete graph $K_{8}$ and the graph $K_{9}$ minus two nonincident edges are rectangle-visibility graphs with $6 n-20$ edges. (See Figure 6 for $K_{8}$; appropriately adding a rectangle in the center produces $K_{9}$ minus two edges.) We use techniques similar to those in [7] to show that a bipartite rectangle-visibility graph on $n \geq 4$ vertices has at most $4 n-12$ edges. This bound is exact for $n=4$. For $5 \leq n \leq 9$ all bipartite graphs on $n$ vertices are rectangle-visibility graphs, and each has at most $n^{2} / 4<4 n-12$ edges. For $n=10$ the bipartite rectangle-visibility graphs with the most edges are $K_{4,6}$ and $K_{5,5}$ minus an edge, each of which has $4 n-16$ edges. Indeed, the $4 n-16$ bound is tight for $7 \leq n \leq 10$, as well as for $K_{4, q}$, and we conjecture that this is the tight bound for $n \geq 7$.

It would be desirable to give a simple characterization of rectangle-visibility graphs, perhaps analogous to the way bar-visibility graphs have been character- 


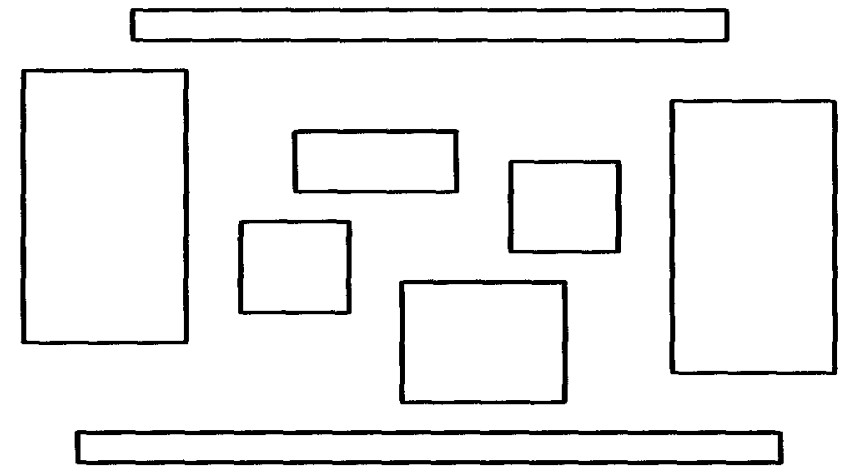

FIGURE 6. A noncollinear rectangle-visibility layout of $K_{8}$

ized, but no characterization has yet been found; neither has the problem been shown to be $N P$-complete. In $[8,12]$ linear-time algorithms are given for deciding bar-visibility. Note also that, while planarity of a graph is decidable in linear time [5], determining whether a graph has thickness at most 2 is $N P$-complete [10]. (See also [1].)

Just as bar-visibility naturally generalizes to rectangle-visibility, so rectanglevisibility can be generalized to visibility between objects in higher dimensions. In [3] a layout is given of the complete graph $K_{42}$, using boxes in 3-space with sides parallel to the axes and visibilities also parallel to the axes. It is easy to realize $K_{m, n}$ as such a "box-visibility" graph (using only one visibility direction) for any $m$ and $n$ as follows: Take a box in $\mathbb{R}^{3}$, cut one side into $m$ horizontal strips, and cut the opposite side into $n$ vertical strips; then each of the $m$ strips sees each of the $n$ strips. Now fatten the strips into boxes and stagger them diagonally to make the graph bipartite. (This observation is due independently to the first author and to the participants of the Workshop on Visibility Representations.)

\section{Main Results.}

In this section we state and discuss the principal theorems of the paper. We first need one or two more definitions.

Noncollinear bar-visibility graphs are those graphs having a bar-visibility layout in which no two segments have endpoints with the same $x$-coordinates. These graphs have been characterized by Luccio, et al., as those planar graphs that are ipo-triangular [9]. A graph $G$ is ipo-triangular if it has a planar embedding that can be transformed into one in which every finite face is a triangle by successive duplications of existing edges. (Graph embeddings with at most one non-triangle face are usually called near-triangulations by graph theorists.) The cycles $C_{n}$, with $n \geq 4$, are a class of graphs that cannot be represented in this way.

Noncollinear rectangle-visibility graphs are those graphs having a rectanglevisibility layout in which no two rectangles have collinear sides. $C_{n}$ does have a noncollinear rectangle-visibility layout for all $n$, as can be generalized from Figure 7. No simple characterization is known for noncollinear rectangle-visibility 
graphs. At the Workshop on Visibility Representations, it was conjectured that every rectangle-visibility graph has a noncollinear representation; for example, Figure 6 shows a noncollinear representation of the largest possible complete graph, $K_{8}$.

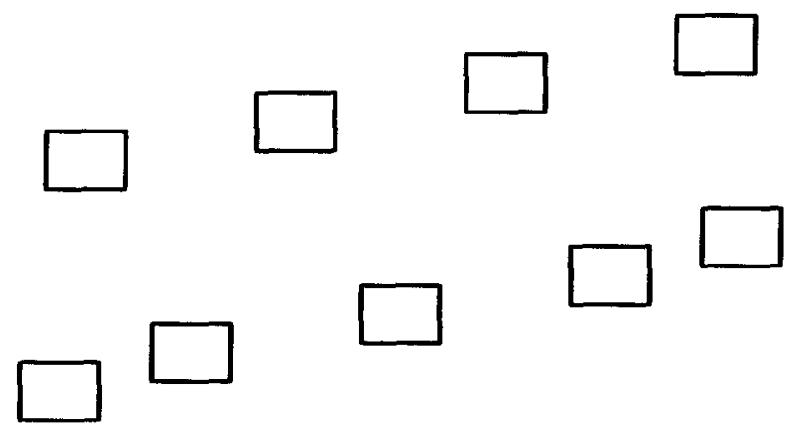

FIGURE 7. A noncollinear rectangle-visibility layout of $C_{9}$

Thus initially it seemed possible that every rectangle-visibility graph had a noncollinear representation. For large $q$, however, the graphs $K_{4, q}$ are likely candidates as counterexamples.

Theorem 1. If $K_{p, q}, p \leq q$, has a noncollinear rectangle-visibility layout and $p \geq 3$, then $q \leq 4$.

Theorem 2. $K_{4,4}$ has no noncollinear rectangle-visibility layout.

The proofs use the Pigeonhole Principle to show that, unless these conditions on $p$ and $q$ are met, there will be unwanted adjacencies in any noncollinear rectangle-visibility layout containing all the edges of $K_{p, q}$.

Figure 2 shows a noncollinear rectangle-visibility layout of $K_{3,4}$, and Figure 1 is easily modified to give a noncollinear layout for $K_{2, q}$; then removing an additional external rectangle gives a layout for $K_{1, q}$. Thus the results of Theorems 1 and 2 are best possible.

Next we consider bipartite representations without concern for collinearity.

Theorem 3. $K_{p, q}, p \leq q$, is a rectangle-visibility graph if and only $p \leq 4$.

The proof of necessity is a proof by contradiction, again making repeated use of the Pigeonhole Principle. The key observation here is that, if we have some rectangle-visibility layout of $K_{p, q}$ with $p, q \geq 5$, then four rectangles, representing two vertices in each side of the bipartition, are forced to have certain positions relative to one another. By a somewhat technical argument, we are then able to show that the relative positions of the remaining rectangles are also forced, leading to a contradiction if $p$ and $q$ are both at least 5 . (This argument can also be used to construct the rectangle-visibility layout of $K_{5,5}$ plus an edge.) The sufficiency of Theorem 5 is demonstrated in Figure 1 above. 
Finally, using techniques similar to those in [7], we have the following two results. Recall that by Euler's formula, a planar (respectively, thickness-two) bipartite graph with $n$ vertices has at most $2 n-4$ (resp., $4 n-8$ ) edges.

Theorem 4. $A$ bipartite rectangle-visibility graph on $n \geq 4$ vertices has at most $4 n-12$ edges.

Corollary 5. If a bipartite graph $G$ with $n$ vertices is a subgraph of a rectanglevisibility graph $G^{\prime}$, then $G$ has at most $4 n-12$ edges.

As noted above, examples indicate that the correct bound should be $4 n-16$ for $n \geq 7$.

Acknowledgments. The authors would like to thank the participants in the Workshop on Visibility Representations, held at the McGill University Bellairs Research Institute, February, 1993, for stimulating conjectures and discussions on these topics, and most especially to thank S.H. Whitesides for significant contributions to the start of the work on Theorem 1. The authors also thank Joe O'Rourke for helpful comments on an earlier version of the paper. Finally, the first author thanks the Department of Computer Science and Engineering at University of California, San Diego, for hosting her as a Visiting Scholar during the 1992-93 academic year, during which time much of the work on this paper was completed.

\section{REFERENCES}

1. T. Andreae, Some results on visibility graphs, Disc. Appl. Math. 40 (1992), 5-18.

2. L. W. Beineke, F. Harary, and J. W. Moon, On the thickness of the complete bipartite graph, Proc. Cambridge Philo. Soc. 60 (1964), 1-5.

3. P. Bose, A. Josefczyk, J. Miller, and J. O'Rourke, $K_{42}$ is a box visibility graph, Tech. Report \#034, Smith College (1994).

4. M. R. Garey, D. S. Johnson, and H. C. So, An application of graph coloring to printed circuit testing, IEEE Trans. Circuits and Systems CAS-23 (1976), 591-599.

5. J. Hopcroft and R. Tarjan, Efficient planarity testing, J. Assoc. Comput. Mach. 21 (1974), 549-568.

6. J. P. Hutchinson, Coloring ordinary maps, maps of empires, and maps of the Moon, Mathematics Magazine 66 (1993), 211-226.

7. J. P. Hutchinson, T. Shermer, and A. Vince, Representations of thickness two graphs, preprint.

8. D. G. Kirkpatrick and S. K. Wismath, Weighted visibility graphs of bars and related flow problems, Lecture Notes in Computer Science (Proc. 1st Workshop Algorithms Data Struct.), vol. 382, Springer-Verlag, 1989, pp. 325-334.

9. F. Luccio, S. Mazzone, and C. K. Wong, A note on visibility graphs, Disc. Math. 64 (1987), 209-219.

10. A. Mansfield, Determining the thickness of graphs is NP-hard, Math. Proc. Camb. Phil. Soc. 93 (1983), 9-23.

11. J. O'Rourke, Art Gallery Theorems and Algorithms, Oxford University Press, N.Y., 1987.

12. R. Tamassia and I.G. Tollis, A unified approach to visibility representations of planar graphs, Disc. and Comp. Geom. 1 (1986), 321-341.

13. S. K. Wismath, Characterizing bar line-of-sight graphs, Proc. 1st Symp. Comp. Geom., ACM (1985), 147-152. 